Hydroécol. Appl. (1993) Tome 5 Vol 1, pp. 29-43

\title{
Surveillance hydrobiologique des centrales nucléaires implantées sur le littoral français
}

\section{Hydrobiological monitoring studies carried out near nuclear power plants built on the sea shore}

\author{
A. Grégoire ${ }^{(1)} n$ R. Gras $^{(2)}$ et M. Khalanski ${ }^{(2)}$ \\ (1) EDF, CNEH, F-73373 Le Bourget du Lac Cedex. \\ (2) EDF, Direction des Etudes et Recherches, Département Environnement, 6, quai Watier, F- \\ 78401 Chatou Cedex
}

Résumé. - Les études de surveillance hydrobiologique du milieu marin menées au droit des centrales électronucléaires révèlent qu'en dehors de l'aspect radiologique l'impact de ces aménagements se présente sous 3 formes principales: thermique, chimique et mécanique. Les organismes aquatiques les plus sensibles sont principalement les jeunes ou, pour les adultes, ceux de petite taille. L'ampleur de la zone concernée par les tâches thermique et chlorée varie de 1 à $10 \mathrm{~km}^{2}$ suivant la configuration des rejets et les caractéristiques du milieu.

Les premiers résultats ont permis de mettre en œuvre des mesures conservatoires pour minimiser les effets dommageables constatés.

Les enseignements tirés permettent de réduire les mesures et analyses systématiques en orientant le programme vers les indicateurs d'impact. Par ailleurs, EDF complète la surveillance par des études spécifiques, de durée limitée, chaque fois qu'un point particulier est à éclaircir sur un site.

Mots-clés. - Centrales nucléaires - Surveillance - Hydrobiologie - Milieu marin.

Abstract. - Except the radiological aspect, hydrobiological monitoring studies carried out near nuclear power plants show three sorts of effects: thermic, chemical and mechanical shocks. The young and small aquatic organisms are mainly affected. The maximum area of the thermal and chlorinated plume, during the whole tide, is about 1 to $10 \mathrm{~km}^{2}$ according to the conditions of the releases and the caracteristics of the site environment.

The first results allow to take measures of conservation to ensure optimum environmental protection.

Lessons drawn from that knowledge allow to decrease the programme of study with the use of impact indicator. In other respects, EDF perfects the monitoring by short-lived specific studies, as often as a special question has to be explained.

Key-words. - Nuclear plant - Monitoring - Hydrobiology - Marine environment. 


\section{INTRODUCTION}

Pour assurer leur refroidissement, les centrales nucléaires côtières prélèvent des quantités importantes d'eau: environ $50 \mathrm{~m}^{3} / \mathrm{s}$ pour une puissance installée de $1000 \mathrm{MWe}$; l'échauffement qui en résulte est de $11^{\circ} \mathrm{C}$ avant dilution. Pendant une partie de l'année, cette eau est chlorée afin d'éviter le développement excessif des salissures biologiques dans les circuits.

Mis à part les rejets radioactifs non abordés dans cette étude, trois types de perturbations de l'écosystème littoral résultent du prélèvement et du rejet de l'eau de refroidissement:

- un impact thermique occasionné par l'échauffement de l'eau,

- un impact chimique dû à la chloration,

- un impact mécanique sur les organismes entraînés dans la prise d'eau et dans le circuit de refroidissement.

\section{PROGRAMMES D'ÉTUDES ET DE SURVEILLANCE}

Afin d'évaluer l'impact du fonctionnement des centrales nucléaires sur l'écosysteme marin, des études hydrobiologiques sont menées par la Direction des Etudes et Recherches d'EDF et par IFREMER (anciennement CNEXO et ISTPM) dans le cadre de conventions passées avec EDF depuis 1976. Ces études ont permis de déterminer l'ordre de gran- deur des perturbations apportées par les centrales et de les minimiser le cas échéant.

Les programmes de surveillance ont pour objet de vérifier que les impacts restent dans les limites prévues par les études antérieures. Ils découlent des dispositions fixées par les arrêtés d'autorisation de rejet donnés par l'Administration. Le programme est établi pour chaque site, pour une durée limitée, en concertation avec le service chargé de la police des eaux et les organismes scientifiques qui ont réalisé les études d'état de référence et d'impact. Sont ainsi définis les paramètres à suivre, l'emplacement des points de prélèvement et la fréquence des analyses à réaliser.

\section{Nature des paramètres}

\section{Hydrologie et chimie}

L'analyse physico-chimique de l'eau porte sur la température, l'oxygène dissous, la salinité et sur les facteurs limitant la production végétale: extinction de la lumière et teneur en sels minéraux nutritifs. Un contrôle des substances générées par la chloration de l'eau est réalisé dans le panache de rejet des centrales qui pratiquent ce traitement.

\section{Organismes d'intérêt halieutique}

Cette partie du programme vise à évaluer les stocks d'organismes d'intérêt commercial inféodés au secteur de plateau continental concerné par l'implantation de la centrale: crustacés (homards, araignées de mer, cre- 
vettes), poissons, mollusques (seiches, calmars).

L'activité de pêche (type, lieu, intensité) est suivie par des enquêtes de même que l'analyse des quantités et de la nature des débarquements pour les principales espèces d'intérêt halieutique. Les formes juvéniles de ces organismes (œufs et larves) sont prises en compte car elles sont plus sujettes à l'entraînement dans les prises d'eau que les adultes.

\section{Pelagos}

L'étude pélagique porte essentiellement sur la faune et la flore planctonique.

Constituant les premiers éléments des chaînes trophiques, le plancton est la source de nourriture des organismes supérieurs notamment ceux exploités commercialement.

L'intérêt de leur étude se justifie d'autant plus que, d'ordinaire, c'est sur ce compartiment que l'impact direct de la centrale est maximal.

L'estimation de la biomasse phytoplanctonique s'effectue par le dosage du pigment photosynthétique commun à tous les groupes d'algues: la chlorophylle «a». Les dinoflagellés responsables des phénomènes d'eaux rouges et les bactéries thermophiles susceptibles de se développer dans les eaux échauffées sont particulièrement surveillés.

L'importance des diverses populations zooplanctoniques est abordée par la mesure globale du poids sec et par le dénombrement des grands groupes. Une attention particulière est apportée aux formes juvéniles des es- pèces d'intérêt halieutique (mollusques, crustacés, poissons).

\section{Benthos}

Les fonds sont généralement colonisés par une faune d'invertébrés qui sont une source de nourriture pour de nombreux poissons et même pour l'homme (certains mollusques et crustacés). L'inventaire consiste en la détermination des espèces ou groupe d'espèces et en l'appréciation de la biomasse.

Sur les côtes à substrat rocheux, les algues qui abondent dans la zone de balancement des marées et sous le niveau des basses mers (fucales, laminaires), sont surveillées en raison de leur sensibilité à toute modification des facteurs physiques et chimiques du milieu. Les programmes comportent sur ces sites un suivi de l'évolution de la couverture algale des fonds.

\section{Stations de prélèvements et mesures}

L'emplacement des points de mesure tient compte de l'hétérogénéité spatiale des facteurs considérés:

- pour le domaine pélagique, deux points (côte et large) sont au moins prospectés sur une verticale;

- pour le domaine benthique, les prélèvements sont effectués le long de deux à trois radiales caractéristiques, de la côte vers le large.

\section{Echéancier}

La fréquence des mesures est fonction d'une part de la variabilité natu- 
relle des paramètres étudiés, d'autre part des connaissances acquises jusque là dans le domaine considéré.

La surveillance démarre environ un an avant la mise en service de la centrale, de façon à réactualiser les données acquises quelques années auparavant et se poursuit pendant plusieurs années après la mise en service industriel des installations (fig. 1). Le programme est révisable après quelques années au vu des résultats obtenus. Ces derniers sont regroupés dans un document, le "Rapport annuel de surveillance de l'environnement", élaboré pour cha- cun des sites et transmis à l'Administration au niveau local et national.

\section{PRINCIPAUX RÉSULTATS \\ ACQUIS LORS DES ÉTUDES DE SURVEILLANCE}

La comparaison de l'état de l'écosystème marin à deux époques différentes (avant et après le démarrage de la centrale) s'étant avérée impossible du fait de la variabilité naturelle interannuelle, IFREMER a été conduit à proposer une nouvelle stratégie

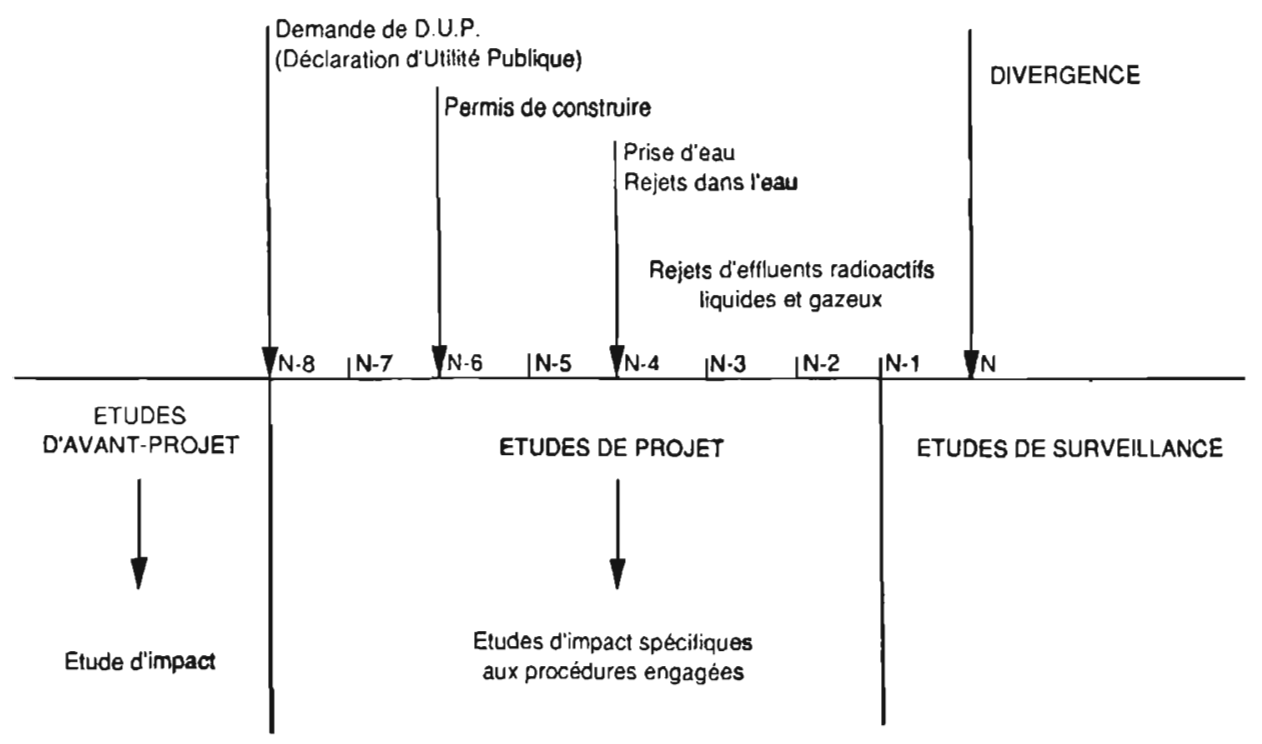

Fig. 1. - Echéancier type des études d'impact.

Fig. 1. - Typical procedure of impact studies. 
d'étude; celle-ci est basée sur la comparaison entre la situation dans la zone la plus proche du rejet supposée perturbée et celle, au même moment, en un point situé hors de la zone impactée.

\section{Impact thermique}

Les résultats acquis jusqu'ici montrent que le secteur concerné par l'impact thermique de la centrale est peu étendu. II n'est perceptible que dans les zones où l'échauffement de l'eau est durable et important (supérieur à $2^{\circ} \mathrm{C}$ ).

Dans ces conditions, on peut constater chez les organismes marins, des modifications d'ordre physiologique et comportemental se traduisant par des changements dans la structure des peuplements, avec remplacement d'espèces. D'une manière générale, la richesse taxonomique a tendance à diminuer.

\section{a) A l'extérieur de la centrale}

\section{- Benthos}

Ce sont les organismes inféodés au fond (benthos) qui sont particulièrement concernés puisqu'en permanence sous l'influence des rejets.

L'expérience de Gravelines montre que seuls les peuplements situés dans la zone proche du rejet (approximativement $5 \mathrm{~km}^{2}$ ) subissent l'influence de l'échauffement de l'eau. Pour le benthos intertidal (entre les limites extrêmes de la marée), la principale modification concerne la substitution d'une espèce de poly- chète par une autre moins thermosensible (Dewarumez, 1982).

Dans les zones les plus chaudes, les cycles de développement de certaines espèces peuvent être accélérés. Ainsi, pour le crustacé Isopode Idotea granulosa, espèce à sa limite méridionale dans la région de la centrale de Paluel, les périodes de recrutement sont différentes à St Valéry-en-Caux (juillet) et à Veulettes (août et octobre). L'impact thermique de la centrale à St Valéry pourrait expliquer la précocité de la reproduction des idotées sur ce site (1 à 2 mois plus tôt qu'à Veulettes) (Ifremer, 1990).

Pour le benthos subtidal (zone en dessous du niveau des plus basses mers), c'est le peuplement à Abra alba qui à Grevelines est altéré par les rejets. Dans ce cas, il semble que ce soit plus l'effet hydrodynamique des rejets qui soit prépondérant; les courant créés par les $240 \mathrm{~m}^{3} / \mathrm{s}$ d'eau échautfée ont, en effet, entraîné à cet endroit, de fortes modifications sédimentologiques. L'élimination des particules et de la vase, entraînent la disparition progressive des espèces vasicoles.

\section{- Plancton}

Dans le domaine pélagique, seuls les micro-organismes sont concernés; les autres semblent ne pas être affectés par l'échauffement de l'eau. Cela peut s'expliquer, notamment, par la faculté que possèdent les organismes pélagiques de grande taille d'éviter les zones où la qualité de l'eau leur est défavorable. 
L'augmentation des températures provoque chez le phytoplancton une stimulation de la croissance. L'augmentation de production primaire peut atteindre $30 \%$ dans la zone échauffée en absence de résidus oxydants produits par la chloration.

Pour ce qui concerne le zooplancton, l'impact thermique peut être considéré comme négligeable. L'étude de l'impact de Gravelines sur les œufs de sole, a montré, par exemple, que les températures létales (de l'ordre de $15^{\circ} \mathrm{C}$ ) sont très éloignées de celles enregistrées au voisinage du rejet, à l'époque de la reproduction de ce poisson (toujours moins de $10^{\circ} \mathrm{C}$ ). Dans ce cas, le seul effet possible de l'échauffement des eaux ne peut être qu'une accélération du développement embryonnaire sans conséquence notable (Lacroix, 1988).

\section{- Bactéries}

L'effet le plus spectaculaire de l'échauffement de l'eau se produit chez les vibrions halophiles. A Gravelines, le facteur de multiplication variait de 13 à 20 en 1982, même en période de chloration (Delesmont et Delattre, 1982; Delesmont et al., 1983). Les concentrations estivales, 100 fois plus élévées qu'en 1979, sont de l'ordre de $10^{5}$ vibrions/l ces dernières années sur une zone d'environ $9 \mathrm{~km}^{2}$. Bien que ces chiffres soient encore inférieurs à ceux relevés dans d'autres secteurs du littoral, il faut signaler leur augmentation régulière d'année en année et surtout le fait que les vibrions ne disparaissent plus en hiver (Daubelcourt et al., 1988).

\section{- Poissons}

A partir des résultats acquis au droit des sites les plus anciens, même avec des échauffements de l'ordre de $10^{\circ} \mathrm{C}$, l'impact thermique sur les poissons reste fort limité. A titre indicatif, on peut préciser qu'au-dessus de $3^{\circ} \mathrm{C}$ d'échauffement environ, les modifications constatées se résument:

- à un changement de la structure des populations au niveau même du rejet et à son voisinage,

- à des migrations saisonnières du fait des effets d'attraction ou de répulsion.

Les poissons préfèrent généralement vivre dans des eaux dont la température leur convient le mieux. Ainsi, est-il fréquent, en particulier en hiver, de voir certains poissons (bars, mulets par exemple), être attirés par les eaux échauffées.

A Gravelines, les mulets fréquentent le canal de rejet en permanence. Les bars, par contre, en sortent lorsque la température dépasse $24^{\circ} \mathrm{C}$ pour les adultes et $27^{\circ} \mathrm{C}$ pour les plus jeunes ( 2 ans); ils y entrent à nouveau à partir de la fin du mois de septembre. L'explication de la présence de ces especes dans le canal de rejet semble être l'effet attractif de l'eau chaude renforcé par la présence d'une nourriture abondante constituée principalement des résidus organiques contenus dans les eaux de lavage des tambours filtrants équipant les prises d'eau de la centrale. La faible croissance des bars du canal, par 
rapport à ceux pêchés en des zones non échauffées, semble montrer que les poissons n'y font là que des passages de courte durée (Brun, 1987).

\section{b) A l'intérieur de la centrale}

Les organismes de petite taille (inférieure à $3 \mathrm{~mm}$ ) entraînés au travers des tambours filtrants des prises d'eau, à l'intérieur des circuits de refroidissement de la centrale subissent des chocs thermiques importants (de l'ordre de $10^{\circ} \mathrm{C}$ ). Néanmoins, l'impact thermique est relativement limité et dépend de la nature des organismes.

Pour le zooplancton, la mortalité résultante est, dans la plupart des cas, inférieure à 10\% (Khalanski, 1984). Seules les mysidacées sont très sensibles à l'échauffement; leur mortalité augmente significativement dès que les températures sont supérieures à $20^{\circ} \mathrm{C}$, c'est-à-dire entre juin et novembre.

En ce qui concerne les bactéries, on ne remarque pas de variations du nombre de germes d'origine fécale entre le canal d'amenée et le canal de rejet. Le choc thermique ne provoque donc ni la destruction ni la prolifération de ces germes. Seule la multiplication des vibrions halophiles est stimulée par l'accroissement des températures; ils sont au moins quatre fois plus nombreux dans le canal de rejet que dans le canal d'amenée.

Enfin, le choc thermique n'affecte en rien le phytoplancton. Au contraire, il stimule la production primaire tant que la température n'excède pas $24^{\circ} \mathrm{C}$.

\section{Impact chimique}

Les rejets chimiques ont 2 origines principales:

- la préparation d'eau déminéralisée qui génère des effluents,

- la chloration pratiquée pour éliminer les salissures biologiques (moules...) qui envahissent les circuits alimentés en eau de mer.

Les effluents de déminéralisation provenant des traitements de floculation-décarbonatation et régénération des résines sont neutralisés puis dilués dans l'eau de refroidissement. Leur absence de toxicité, vérifiée par des tests toxicologiques (Khalanski, 1989) s'explique par leur nature minérale (sulfate, carbonate, hydroxyde ferrique, chlorures, sodium, calcium) et leur grande dilution.

Les rejets de chlore sont donc seuls susceptibles de créer quelques dommages au milieu marin.

Dans les centrales thermiques côtières, la chloration est pratiquée par injection d'hypochlorite au droit des stations de pompage. En Mer du Nord et en Manche, lieu d'implantation des centrales nucléaires de Gravelines, Penly, Paluel et Flamanville, le procédé de lutte contre la fixation des salissures biologiques (moules principalement) dans les circuits, nécessite une chloration en continu dès que la température des eaux dépasse $10^{\circ} \mathrm{C}$. Dans le but de protéger au mieux le milieu marin, des études d'optimisation ont permis à Gravelines de ramener le niveau minimal de chlore efficace de $1 \mathrm{mg} / \mathrm{l}$ à $0,8 \mathrm{mg} / \mathrm{l}$. 
Les produits oxydants générés par la chloration de l'eau de mer sont essentiellement du brome libre et du brome combiné (hypobromites et bromamines). C'est la toxicité de ces produits qui rend compte de l'effet antisalissures. Les espèces oxydantes présentent l'avantage de disparaître rapidement par réactions chimiques; seuls persistent à plus long terme des composés organohalogénés, dont le principal est le bromoforme, mais qui sont très peu toxiques.

Les effets sont variables suivant les groupes d'organismes concernés. L'exemple de Gravelines fournit des enseignements particulièrement intéressant en la matière.

Chez les bactéries, la chloration entraîne une mortalité oscillant entre 30 et $50 \%$ suivant l'espèce, à la sortie du canal de rejet. Cet effet s'atténue très vite après la sortie du canal. Le potentiel hétérotrophe est, de plus, inhibé (Daubelcourt, 1987).

La chloration affecte également la production primaire et la biomasse phytoplanctonique (Bourgade et Ramade, 1981). Dans le canal de rejet, la chlorophylle est détruite à raison de 25 à $50 \%$ par rapport à celle du canal d'amenée. La production primaire peut chuter, dans une proportion de $95 \%$.

La diminution du nombre de cellules phytoplanctoniques ne peut être due qu'à l'effet du chlore puisqu'elle ne se produit pas hors période de chloration. II existe aussi un déficit en chlorophylle "a", dans le panache, au plus égal à la quantité de chlorophylle ayant transité dans les circuits de réfrigération. Cela montre que l'impact de la chloration est ressenti surtout pendant le transit et que les effets sont faibles, voire nuls à l'extérieur du canal de rejet, dans la zone où les oxydants résiduels totaux ne subsistent qu'à l'état de traces.

Au droit des centrales de Paluel et Flamanville, du fait des rejets au large en profondeur, la rapidité de la dilution masque presque totalement ce phénomène.

La chloration a un effet beaucoup moins prononcé mais plus irrégulier sur le zooplancton. Globalement, elle entraîne un accroissement de la mortalité inférieur à 10\%. Parmi le méroplancton, ce sont les larves d'annélides et de décapodes qui sont les plus résistantes. Sur les œufs et les larves de poissons, l'effet de la chloration est faible par rapport à l'effet mécanique. Pour l'holoplancton, c'està-dire principalement les copépodes, le chlore n'est en général plus toxique aux concentrations rencontrées dans le canal de rejet, hormis pendant de rares périodes pour cause de mauvais mélange de la solution d'hypochlorite. L'effet de la chloration sur le zooplancton est donc limité au transit (Khalanski, 1985).

De manière générale, on peut considérer qu'au droit des rejets, l'impact chimique n'est décelable que si les teneurs en oxydants résiduels dépassent $0,05 \mathrm{mg} / \mathrm{l}$. L'étendue de cette zone est, à Gravelines, très inférieure au $\mathrm{km}^{2}$. 
En ce qui concerne le bromoforme, il n'est pas toxique aux concentrations mesurées dans le canal de rejet. Le problème de sa bioaccumulation a pourtant été examiné en raison de sa relative stabilité chimique. Les dosages de bromoforme effectués dans la chair et le foie de bars ont montré que les facteurs de concentration calculés, par rapport à l'eau d'élevage, sont respectivement de 3 et 8 . L'élimination du bromoforme est cependant rapide; un mécanisme de désintoxication intervient très rapidement puisque, dans un milieu sans chiore, après 3 jours, cet élément se stabilise à des valeurs de l'ordre de 1 dans la chair et de 3 dans le foie. En 1981, les concentrations dans les organismes marins sont restées inférieures à $50 \mu \mathrm{g} / \mathrm{kg}$ ce qui exclut à la fois tout risque de toxicité pour les organismes et tout risque sanitaire lié à leur consommation. Depuis 1981, les teneurs en bromoforme mesurées chaque année, dans l'eau, permettent de supposer que l'augmentation des concentrations dans les organismes, en période de chloration, a été réduite au cours des dernières années.

\section{Impact mécanique: prises d'eau et intérieur de la centrale}

Les organismes entraînés dans les circuits d'eau sont soit des organismes planctoniques (phytoplancton, zooplancton), soit des organismes à capacité natatoire réduite du fait de leur petite taille (petits poissons, alevins, juvéniles), de leur morphologie ou de leur mauvais état physiologique (animaux affaiblis par la maladie, la fatigue, la reproduction...). Très peu d'organismes de taille supérieure à $10 \mathrm{~cm}$ sont ainsi concernés.

En fonction de leur taille, deux cas se présentent, suivant que ces organismes peuvent traverser ou non les mailles ( 3 à $4 \mathrm{~mm}$ de vide) des grilles des tambours filtrants.

Les organismes dont la taille est supérieure à $4 \mathrm{~mm}$ sont plaqués contre les tambours filtrants où le système de nettoyage sous pression peut les endommager. Le taux de mortalité varie suivant les espèces. Certaines, comme les anguilles sont très résistantes alors que d'autres, comme les harengs et les sprats, sont très fragiles (près de $100 \%$ de mortalité). Les crevettes et les poissons plats ont, quant à eux, une résistance moyenne (Victor-Baptiste, 1984).

Les dispositifs de dissuasion s'avérant inefficaces, pour minimiser l'impact des prises d'eau, les actions se sont orientées vers la récupération des organismes sur les tambours filtrants ou dans les chambres d'eau. On évalue les prises à quelques dizaines de tonnes par tranche et par an à Gravelines et Paluel. Outre les poissons, les principaux groupes concernés sont les méduses, les cténaires (groseilles de mer) et les crevettes (Travade, 1987).

Les organismes dont la taille est inférieure à $4 \mathrm{~mm}$ traversent les filtres et circulent dans les circuits de réfrigération où ils sont soumis à des chocs chimiques, thermiques et mécaniques (chocs contre les parois et modifications de pression) relativement intenses mais de courte durée. 
Là encore, les dommages varient suivant les groupes. Les œufs et larves de poissons, ainsi que le phytoplancton (jusqu'à $80 \%$ de mortalité) sont parmi les plus sensibles (Woerling, 1983).

En tout état de cause, la quantification de l'impact des prises d'eau, à long terme, est très difficile à appréhender car, d'une part les mortalités concernent surtout les juvéniles qui sont eux-mêmes soumis dans le milieu à une très forte sélection naturelle, d'autre part les stocks de la plupart des espèces capturées sont mal connus.

\section{Action conjuguée des effets mécaniques, thermiques, chimiques}

Pour la majorité des organismes, la résultante des impacts est la somme de chacun pris séparément. Ainsi, pour les copépodes et les larves nauplii de cirripèdes, les larves d'annélides et de crabes, les mortalités globales après avoir subi tous les effets du transit, sont comprises entre 20 et $30 \%$. Pour les mysidacées, la conjugaison de ces trois impacts induit un très faible taux de survie (inférieur à $10 \%$ ).

Pour d'autres organismes, les effets se surajoutent mais parfois se contrecarrent. Quelques exemples illustrent ces faits:

- Pour le phytoplancton, les effets sont antagonistes: le chlore tue les cellules végétales mais l'échauffement stimule leur multiplication; il de- meure, toutefois, un déficit permanent en chlorophylle sur le site. De même, pour les vibrions halophiles, le chlore limite la prolifération des germes dans la zone la plus échauffée.

- Pour les œufs de poissons, sole en particulier, les études montrent que la mortalité totale est supérieure à la somme des mortalités induites par chaque effet produit séparément puisque l'augmentation de la mortalité sous l'action cumulée des trois effets est d'environ $20 \%$. II a été constaté que les chocs thermiques et chimiques ne sont traumatisants que s'ils se produisent simultanément.

\section{PERSPECTIVES D'ÉVOLUTION DES PROGRAMMES DE SURVEILLANCE}

Sur la base de l'expérience acquise, la conception d'EDF est de suivre, dans le champ proche, un nombre limité de paramètres indicateurs de l'évolution du milieu et susceptibles de permettre de détecter les anomalies éventuelles notables engendrées par la centrale.

Lorsque l'évolution d'un processus, dans lequel le fonctionnement de la centrale est impliqué, mérite d'être suivi (par exemple la pullulation de vibrions halophiles), il est clair que le programme de surveillance doit en tenir compte; des études complémentaires sont alors engagées pour mieux cerner cette évolution. Des études spécifiques, de durée limitée, sont 
ainsi réalisées chaque fois qu'un point particulier est à éclaircir sur un site. Elles fournissent des données précises sur les perturbations physicochimiques et biologiques observables localement: échauffement, teneur en oxydant résiduel, taux de mortalité du plancton au rejet, quantité de poissons capturés aux prises d'eau.

En revanche, lorsque les connaissances acquises ont abouti à des conclusions claires confirmées par des années de mesures sur site, la question de la pertinence de la poursuite de ces mesures se pose. La problématique de l'optimisation du programme de surveillance repose a priori sur quelques criteres de base:

- l'évolution du programme doit intégrer les connaissances acquises,

- les données collectées doivent permettre de caractériser localement un impact identifié,

- certains impacts ne peuvent pas être mesurés mais une évaluation quantitative peut être obtenue par la mise en cuvre d'outils théoriques, les modèles de simulation.

Concernant le premier point, il est clair que le travail d'interprétation des données réalisé par IFREMER et par EDF a permis de préciser localement les limites de l'impact des centrales. Lorsqu'aucune évolution significative n'est mise en évidence malgré plusieurs années d'investigations, l'étude de surveillance pourrait alors n'être réalisée que tous les 3 ou 5 ans. Une autre solution est de réaliser alternativement, suivant les années, des campagnes dites "lourde" ou "légère" (cas actuel du site de Paluel).
Le second point pose la question des "indicateurs d'impact"; certaines mesures ne fournissent aucune information sur les impacts identifiés ou sont redondantes avec d'autres grandeurs mesurées.

Le dernier point est certainement le plus difficile à aborder; il s'inscrit en effet dans le contexte de la signification de la mesure en écologie. Le problème de la signification statistique des écarts entre mesures ne peut être éludé: détecter une différence de $5 \%$ sur la biomasse planctonique entre deux stations implique par exemple un effort d'échantillonnage pratiquement irréalisable. Des questions sont d'autre part soulevées par l'absence de frontière des processus écologiques: peut-on, à partir d'un lot de données collectées localement, tirer des conclusions sur un phénomène qui se développe à plus grande échelle spatiale et qui comporte des éléments n'intervenant pas sur le site de mesure?

Intégrer un programme de mesures locales dans un contexte spatial plus vaste, dans une étude plus diversifiée permet de résoudre en partie ces difficultés. Le projet de création d'un comité d'étude des ressources halieutiques en Gironde, placé sous l'autorité préfectorale, laisse augurer d'une évolution des pratiques dans ce sens.

De même, lorsque le niveau de connaissance des processus écologiques le permet, le recours à la modélisation numérique est le moyen de mieux approcher l'incidence de modifications d'une ou plusieurs compo- 
santes sur l'ensemble du système (Agoumi, 1985). La mise en œuvre de modeles thermiques a permis de réduire le réseau de surveillance thermographique mis en place sur le site de Gravelines. Mais les codes de calcul de courants et de dilution élaborés à cette occasion ne se limitent pas aux seuls aspects thermiques; ils ont des applications plus générales dans des domaines variés comme la dilution des effluents radioactifs ou chimiques, la pollution par les hydrocarbures ou les problèmes sédimen- tologiques. Dans le domaine des peuplements, les modèles planctoniques sont d'ores et déjà appliqués à l'évaluation d'impact comme cela a été fait à Gravelines (Menesguen, 1984 ; Gosse et Agoumi, 1985). Le recours aux modèles de dynamique de population est également envisageable. La modélisation ne peut pas se passer de mesures mais il s'agit alors d'un programme spécifique portant sur des paramètres très particuliers avec une fréquence adaptée au pas de temps du calcul.

\section{CONCLUSION}

L'expérience fondée sur une douzaine d'années d'étude montre que la quantité d'eau transitant dans les installations, l'importance de l'échauffement et la nature des éléments chimiques rejetés sont les principaux facteurs discriminants de l'impact du fonctionnement des centrales électronucléaires sur le milieu aquatique.
Dans les zones échauffées de $2^{\circ} \mathrm{C}$ et plus, il peut se produire des modifications de la flore et de la faune, du fait du remplacement d'espèces d'eau froide et bien diversifiées par des espèces plus thermophiles et moins nombreuses. En outre, le cycle de développement de certains taxons peut être modifié et, parfois, aboutir à la prolifération d'une population comme les vibrions halophiles à Gravelines. Les poissons ont, quant à eux, une réponse essentiellement comportementale d'attraction ou de répulsion qui les fait rechercher ou fuir les zones les plus échauffées.

L'impact des rejets chimiques résulte de l'utilisation de chlore pour éviter la fixation des moules dans les prises d'eau. Compte tenu des faibles concentrations en oxydants résiduels contenus dans les rejets $(<0,05 \mathrm{mg} / \mathrm{l})$, l'effet toxique se limite pratiquement à une inhibition de la photosynthèse planctonique et à une réduction des germes bactériens. Le secteur concerné est en général inférieur au $\mathrm{km}^{2}$.

L'influence du fonctionnement d'une centrale nucléaire sur les organismes aquatiques est, pour la plupart d'entre eux, la somme de chaque type d'impact pris séparément. Néanmoins, dans certains cas, les effets de l'échauffement de l'eau et de la chloration se surajoutent (action sur le zooplancton) alors que dans d'autres ils sont antagonistes (cas des vibrions halophiles et du phytoplancton).

En tout état de cause, même au droit de Gravelines, la plus importante centrale nucléaire de France avec 


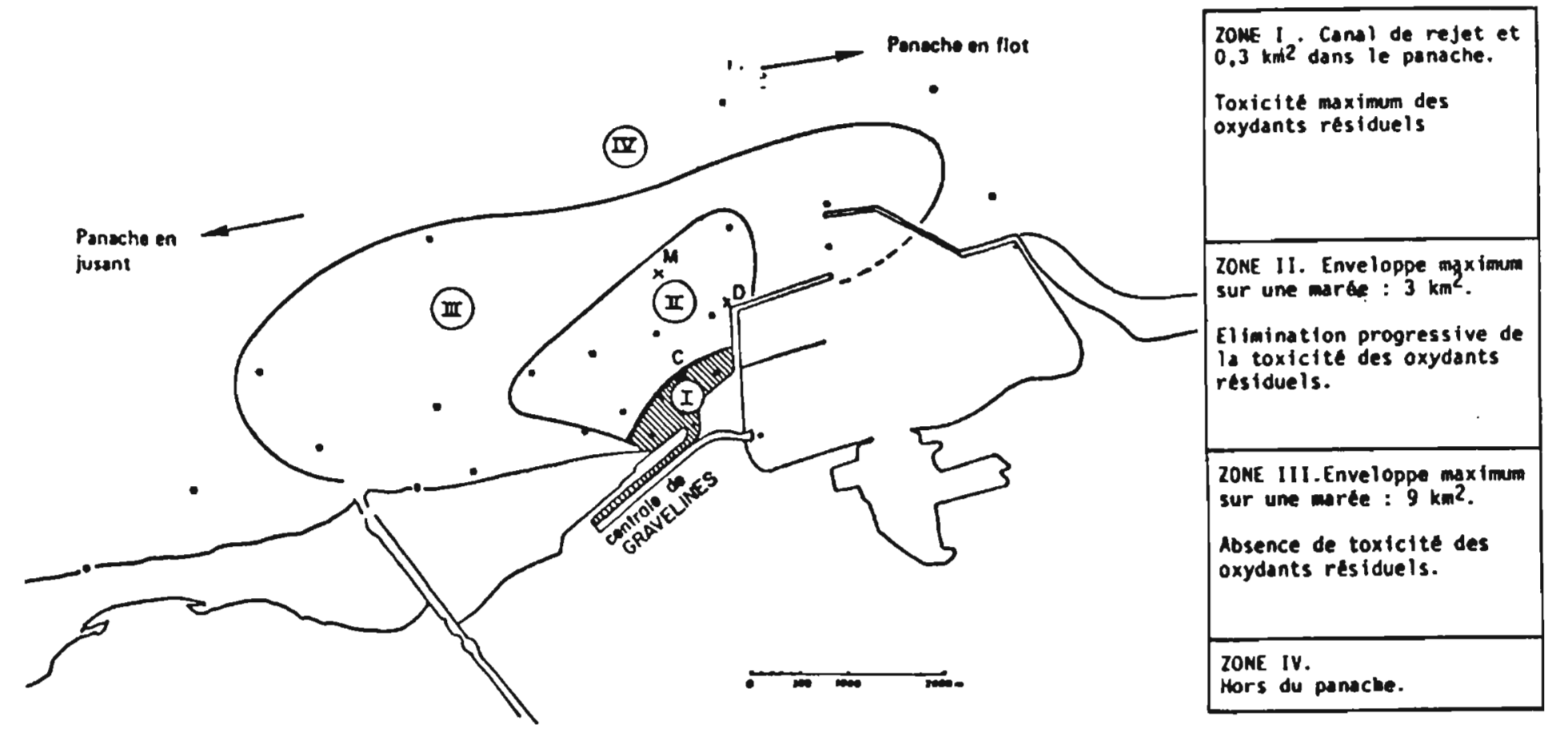


$6 \times 900 \mathrm{MW}$, la zone impactée ne représente qu'une enveloppe globale de $9 \mathrm{~km}^{2}$ compte tenu du balancement des marées (fig. 2).

Des mesures conservatoires sont, de plus, mises en œuvre pour minimiser les effets sur l'environnement. Ce sont:

- pour limiter l'impact thermique, une conception appropriée des ouvrages de prise et de rejet d'eau permettant d'assurer la meilleure dilution possible;

- pour limiter limpact chimique, l'optimisation des quantités de chlore injecté destinée à abaisser la concentration minimale de chlore efficace;

- pour limiter l'impact mécanique, l'aménagement de la configuration de la prise d'eau ou la possibilité de remplacer, dans certains cas, les jets à haute pression par des jets à basse pression afin de décoller, sans trop de dommages, les organismes des filtres rotatifs.

Compte-tenu des connaissances acquises en plusieurs années de surveillance, et après une synthèse des résultats obtenus, les programmes sont actualisés périodiquement pour chaque site. Les enseignements tirés permettent de réduire les mesures et analyses systématiques en orientant le programme vers les indicateurs d'impact. Ces données permettent, au-delà de l'aspect réglementaire, une validation des résultats des études d'impact et une base d'amélioration des outils prévisionnels mis en œuvre. Elles sont également des éléments clefs pour optimiser des conditions de fonctionnement vis-à-vis du respect de l'environnement.

\section{REFERENCES}

Agoumi A., 1985. Modélisation de l'écosystème pélagique en Manche. Thèse de Docteur ès Sciences, Université Pierre et Marie Curie, Paris 6.

Bourgade E., Ramade A., 1981. Incidence des chocs mécaniques et de la chloration sur le phytoplancton à la centrale de Gravelines. Rapport EDF/DER. $\mathrm{HE} / 31.81 .6$.

Brun A., 1987. Transfert dans la chaine alimentaire des organismes entrainés dans les circuits de prise de la centrale nucléaire de Gravelines. Mémoire de fin d'études. Spécialisation halieutique. ENSA Rennes, $58 \mathrm{pp}$.

Daubelcourt C., 1987. Etude microbiologique et des produits de chloration sur l'eau de mer à Gravelines. Rapport d'avancement. Rapport EDF/DER.HE/ 32.87.25, $10 \mathrm{pp}$.

Daubelcourt C., Delattre J.M., Delesmont R., 1988. Etude des résidus chlorés de l'eau de mer à Gravelines et conséquences microbiologiques. Rapport d'avancement EDF/DER HE/31.88-33, $14 \mathrm{pp}$.

Delesmont R., Delattre J.M., 1982. Etudes microbiologiques des effets thermiques et de la chloration en bord de mer (Gravelines). Rapport EDF/DER HE/31.82.36, $66 \mathrm{pp}$.

Delesmont R., Delattre J.M., Lepercq E., 1983. Incidence du fonctionnement de la centrale de Gravelines sur l'activité bactérienne marine. Vibrions halophiles. Rapport établi pour EDF/DER par l'Institut Pasteur de Lille, $38 \mathrm{pp}$.

Dewarumez J.M., 1982. Exemple d'impact sur le milieu intertidal de la mer du 
Nord: le complexe "Avant-port ouest de Dunkerque - centrale nucleaire de Gravelines " - Poster présenté au $17^{\circ}$ EMBS. Brest.

Gosse Ph. et Agoumi A., 1985. Evaluation par simulation numérique de l'impact de la centrale de Gravelines sur le phytoplancton en Mer du Nord. Rapport EDF/DER HE/31.85.34.

IFREMER 1990. Surveillance écologique et halieutique, Site de Paluel, Année 1989, Dro EL, 90.28, 115 p.

Khalanski M., 1984. Données sur la survie du zooplancton ayant transité dans le circuit de refroidissement de la centrale de Gravelines. Rapport EDF/DER $\mathrm{HE} / 31.84 .49,46 \mathrm{pp}$.

Khalanski M., 1985. Impact de la chloration sur la qualité de l'eau et le plancton. Bilan des études réalisées sur le site de Gravelines de 1979 à 1983. Rapport EDF/DER HE/31.85.09, 70 pp.

Khalanski M., 1989. Impact des rejets résultants de la décarbonatation de l'eau brute au CPN de Gravelines. Rapport EDF/DER HE/31.89.39.
Lacroix N., 1988. Effects of thermal discharges on marine resources. In: $/ /^{\theta}$ colloque franco-japonais d'Océanographie, 3-13 octobre 1988 Shimizu, Japon, $7 \mathrm{pp}$.

Menesguen A., 1984. Evaluation par modélisation numérique de l'impact planctonique dans le panache de rejet de la centrale de Gravelines. Rapport interne IFREMER.

Travade F., 1987. Aspiration d'organismes aux prises d'eau des centrales. RGN, 1, janv.-fév., 59-62.

Victor-Baptiste $F$, 1984. Elude qualitative et quantitative des organismes entrainés dans la centrale de Gravelines. Essai de quantification de l'impact global de la centrale: application au hareng. Rapport EDF:DER HE/31.85.38, $441 \mathrm{pp}$.

Woerling D., 1983. Etude expérimentale de l'impact de la centrale de Gravelines (Nord) sur les œufs et larves de la sole (1982-1983). Rapport I.S.T.P.M.

Signification des sigles:

CNEXO: Centre National de l'Exploitation des Océans

EDF : Électricité de France

IFREMER: Institut Français de Recherche pour l'Exploitation de la Mer

ISTPM: Institut Scientifique et Technique des Pêches Maritimes 